\title{
Thermodynamical Study of the Thermoelectric Effect for Magnesium Silicide ${ }^{\dagger}$
}

\author{
Z. H. Zhu ${ }^{\ddagger}$ and Chaoyuan Zhü, \\ Institute of Atomic and Molecular Physics, Sichuan University, Chengdu 610065, China, and Department of \\ Applied Chemistry, Institute of Molecular Science and Center for Interdisciplinary Molecular Science, \\ National Chiao-Tung University, Hsinchu 300, Taiwan
}

Received: May 19, 2007; In Final Form: August 3, 2007

\begin{abstract}
The thermoelectric effect of magnesium silicide is studied by using a thermodynamical method in the presence of an electric field. The thermoelectric potential is evaluated from the partial derivative of free energy with respect to charge in which the free energy is calculated at the B3LYP/6-31G(d,p) level of density functional theory. This free energy is also utilized to determine the average dipole moment from which the polarizability, $\alpha$; molar polarization, $\Psi$; and dielectric constant can be computed. The present calculation for the dielectric constant $(\sim 24-20)$ is in very good agreement with the experimental value (20). This accurate dielectric constant can be used to derive the relation of the thermoelectric potential with respect to temperature, from which the thermoelectric power or the Seebeck coefficients are calculated. The present result shows good agreement with experiment measurement for the Seebeck coefficients. In comparison, that calculation from the energy band structure theory is far off from the experimental values.
\end{abstract}

\section{Introduction}

Material with thermoelectric energy conversion has attracted a great deal of attention for its applications to temperature measurement; cooling of laser modules; computer chip coolers; and particularly, for the recovery of exhaust heat sources to generate electric power and to reduce global warming due to the greenhouse effect. For thermoelectric energy conversion to be highly efficient, the thermoelectric conversion efficiency, defined by a dimensionless figure of merit, ZT, which is relevant to $10 \%$ conversion efficiency, must be greater than unity. The figure of merit is defined by

$$
\mathrm{ZT}=\frac{S^{2} \sigma T}{k}
$$

where $S$ is called Seebeck coefficient, $\sigma$ is the electrical conductivity, $k$ is the thermal conductivity, and $T$ is temperature. The ideal thermoelectric material would have a large $S$ and small $k$, like an insulator, but would also have a high $\sigma$, like a metal.

Magnesium silicide $\left(\mathrm{Mg}_{2} \mathrm{Si}\right)$, an alkali monosilicide that has the space group $F m 3 m$ with crystal parameter 6.351 , is an inexpensive, promising, n-type semiconductor material at temperatures ranging from 500 to $773 \mathrm{~K}^{1-6}$ for its narrow-band gap of $0.78 \mathrm{eV}^{16}$, and is notable for the abundance of its constituent elements in the earth's crust and its lack of toxicity. Its density is $1.88 \mathrm{~g} / \mathrm{cm}^{3}$. ${ }^{7}$ The Seebeck coefficient is conventionally based on the calculation of the carrier motion of the energy band, which includes two parts: diffusion thermoelectric power and phonon drag power. ${ }^{8}$ For example, for an n-type

\footnotetext{
$\dagger$ Part of the "Sheng Hsien Lin Festschrift".

* To whom correspondence should be addressed. E-mail: cyzhu@ mail.nctu.edu.tw.

$\doteqdot$ Sichuan University.

$\S$ National Chiao-Tung University.
}

semiconductor, the diffusion thermoelectric power is defined by

$$
\alpha_{n}=-\frac{k_{\mathrm{B}}}{q}\left[\frac{3}{2}+\ln \frac{2\left(2 \pi m_{n}^{*} k_{\mathrm{B}} T\right)^{3 / 2}}{n h^{3}}\right]
$$

where $k_{\mathrm{B}}$ is the Boltzmann constant, $h$ is the Planck constant, $q$ is the electron charge, $m_{n}^{*}$ is the effective electron mass, and $n$ is the effective electron concentration. Accurate calculations based on eq 1 cannot be easily performed.

The present work suggests another alternative way to study the thermoelectric effect based on thermodynamic theory for the systems that are in equilibrium with an electric field. The first step is to calculate the Helmholtz free energy and dipole moment at different electric fields; then it is possible to obtain the polarizability, distortion polarizability, and dielectric constant for $\mathrm{Mg}_{2} \mathrm{Si}$. Thermodynamics can be considered as an exact theory in which all microscopic quantities are systematically averaged out so that it is possible to perform a more accurate calculation. However, some of experimental data are needed: for instance, the density of solid $\mathrm{Mg}_{2} \mathrm{Si}$.

In Section II, the thermodynamic method in the presence of an electric field is briefly introduced. Section III is concerned with the thermodynamical study of the free energy, polarizability, and dielectric constant for solid $\mathrm{Mg}_{2} \mathrm{Si}$ by combining the molecular structure calculation of density functional theory. The thermoelectric effect and calculation of the Seebeck coefficient is presented using three different methods in Section IV. Concluding remarks are given in Section V.

\section{Thermodynamic Method}

For the self-contained purpose of the present study, we briefly review the thermodynamic method in the presence of an electric field by using Samoluoviqi's reference book ${ }^{9}$ and its notations. Following the Gibbs phase rule, the equilibrium state for a onecomponent homogeneous system is defined by two intensive 
TABLE 1: The Free Energy, $F$ (au), with Electric Field (au) and Temperature (K) (B3LYP/6-311G(p,d))

\begin{tabular}{lccccccc}
\hline & \multicolumn{7}{c}{ field } \\
\cline { 2 - 7 } \multicolumn{1}{c}{ temp } & 0.00 & 0.01 & 0.02 & 0.03 & 0.04 & 0.05 & 0.06 \\
\hline 300 & 689.6559962 & 689.6762975 & 689.7142636 & 689.7700045 & 689.8437878 & 689.9347311 & 690.0422010 \\
400 & 689.6682658 & 689.6886129 & 689.7266498 & 689.7826513 & 689.8564908 & 689.9474521 & 690.0546827 \\
500 & 689.6810849 & 689.7014751 & 689.7395853 & 689.7955607 & 689.8697470 & 689.9607214 & 690.0677121 \\
600 & 689.6943451 & 68.7147797 & 689.7529623 & 689.8090536 & 689.8834422 & 689.9744318 & 690.0811824 \\
700 & 689.7077295 & 689.7284523 & 689.7667074 & 689.8229167 & 689.8975060 & 689.9885107 & 690.0950201 \\
800 & 689.7219110 & 689.7424410 & 689.7807660 & 689.8370927 & 689.9118850 & 690.0029037 & 690.1092450 \\
dipole /debye & 2.8764 & 7.3629 & 11.8296 & 16.3347 & 20.8627 & 25.3297 & 29.6381
\end{tabular}

quantities; for instance, temperature and pressure, temperature and electric field $(T, \epsilon)$, etc. For the present study, we choose $T$ and $\epsilon$ as the independent intensive quantities. Thermodynamical equations are given by

$$
\mathrm{d} F=-S \mathrm{~d} T-P \mathrm{~d} \epsilon
$$

and

$$
\mathrm{d} G=-S \mathrm{~d} T-P \mathrm{~d} \epsilon
$$

from which the average dipole moment, $\mu$, is defined by

$$
\mu=-\left(\frac{\partial F}{\partial \epsilon}\right)_{T}=-\left(\frac{\partial G}{\partial \epsilon}\right)_{T}
$$

where $F, G$, and $S$ stand for free energy, free enthalpy, and entropy, respectively. All quantities, including the average dipole moment $\mu$, are given in atomic units (au). At a given temperature, the average dipole moment in eq 4 is equal to the polarizability $\alpha$ by unit electric field. Thus, the molar polarization can be obtained as

$$
\psi=\frac{4 \pi}{3} N_{0} \alpha=2.522 * 10^{24} \alpha
$$

where polarizability $\alpha$ is the sum of the electronic polarizability, $\alpha_{E}$; atomic polarizability, $\alpha_{A}$; and orientation polarizability, $\alpha_{0}$;

$$
\alpha=\alpha_{D}+\alpha_{0}=\alpha_{E}+\alpha_{A}+\alpha_{0}
$$

where $\alpha_{D}=\alpha_{E}+\alpha_{A}$ is called as the distortion polarizability.

The distortion polarizability can be calculated by

$$
\mu=\alpha_{\mathrm{D}} \epsilon_{\mathrm{in}}=\left(\alpha_{\mathrm{E}}+\alpha_{\mathrm{A}}\right) \epsilon_{\mathrm{in}}
$$

where $\epsilon_{\text {in }}$ is the inner electric field and given by

$$
\epsilon_{\mathrm{in}}=\epsilon-\epsilon_{\mathrm{pol}}+\epsilon_{\mathrm{ca}}
$$

in which $\epsilon$ represents the external field, $\epsilon_{\mathrm{pol}}$ is the polar electric field, and $\epsilon_{\mathrm{ca}}$ is the cavity electric field. From the relation of dipole moment with the inner electric field, $\epsilon_{\text {in }}$, or approximately with the external field, $\epsilon$, distortion polarizability can be evaluated by eq 7 , and thus, the molar distortion polarization can be obtained as

$$
\psi_{\mathrm{D}}=\frac{4 \pi}{3} N_{0} \alpha_{\mathrm{D}}=\left(2.522 \times 10^{24}\right) \alpha_{\mathrm{D}}
$$

\begin{tabular}{|c|c|c|c|}
\hline $\begin{array}{l}T \\
\mathrm{~K}\end{array}$ & $\begin{array}{l}\text { the functions } \\
\text { with elect }\end{array}$ & $\begin{array}{c}\alpha \\
10^{24} \mathrm{~cm}^{3}\end{array}$ & $\begin{array}{c}\psi \\
\mathrm{cm}^{3} \mathrm{~mol}\end{array}$ \\
\hline 300 & -8771 & & \\
\hline 40 & 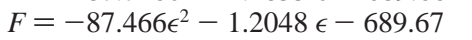 & & \\
\hline 50 & $F=-$ & 26 & DJ. \\
\hline 600 & $\epsilon-689.699$ & 26 & 65.70 \\
\hline 700 & $639 \epsilon-689.71$ & 25 . & 65.469 \\
\hline 800 & $F=-86.84 \epsilon^{2}-1.2683 \epsilon-689.72$ & 25.961 & 65.473 \\
\hline
\end{tabular}

Before we go to the next section, it would be better to present the corresponding relations of the atomic unit: length is 1 au $=1 \mathrm{Bohr}$, charge is $1 \mathrm{au}=1.602189 \times 10^{-19} \mathrm{C}$, energy is 1
TABLE 2: The Functions of Free Energy with Respect to theElectric Field $(\epsilon)$, Polarizability $(\alpha)$ and Molar Polarization $(\psi)$ for $\mathrm{Mg}_{2} \mathrm{Si}$

$\mathrm{au}=4.3598 \times 10^{-11} \mathrm{erg}$, electric field is $1 \mathrm{au}=5.142258 \times$ $10^{9} \times 1 / 300 \mathrm{cgse} / \mathrm{cm}$, and dipole moment 1 Debye $=10^{-18}$ cgse-cm.

\section{Free Energy, Polarizability, and Dielectric Constant of $\mathrm{Mg}_{2} \mathrm{Si}$}

A. The Function of Free Energy, $F$ (au), with Electric Field (/au) and Temperature (K). On the basis of studying the dissociation energy of the ground state $\mathrm{OS}_{2}$ molecule at the B3LYP/6-311G(p,d) level of density functional theory, ${ }^{17}$ we know that the same level of quantum mechanical method can be suitably used for the structure calculation of $\mathrm{Mg}_{2} \mathrm{Si}$. The detailed electronic structure calculation is presented in ref 17. In the present work, we simply report calculation results of electronic energy, thermodynamic energy, thermodynamic entropy, and dipole moment for $\mathrm{Mg}_{2} \mathrm{Si}$ at various temperatures: $300,400,500,600,700$, and $800 \mathrm{~K}$ and at various electric fields: $0.00,0.01,0.02,0.03,0.04,0.05$, and $0.06 \mathrm{au}$, respectively. The thermodynamic free energy of $\mathrm{Mg}_{2} \mathrm{Si}$ is given as follows,

$$
F=U-T S
$$

where $U$ is the ground state electronic energy. The results for $F$ are listed in Table 1. To calculate the average dipole moment, we must fit the data in Table 1 into the certain analytical function, and as the example, the function of free energy with respect to the electric field for $\mathrm{Mg}_{2} \mathrm{Si}$ at $600 \mathrm{~K}$ is fitted as follows:

$$
F=-87.168 \epsilon^{2}-1.2353 \epsilon-689.699
$$

Similarly, all fitted functions for the different temperatures are summarized in Table 2. The present fitting functions well represent the original data. As an example, we demonstrate it in Figure 1 for the cases of temperature at 400, 600, and $800 \mathrm{~K}$.

B. Polarizability $(\alpha)$ and Molar Polarization $(\psi)$. Since we get the analytical function of free energy with respect to the electric field, we can use eq 4 to compute the average dipole moment, $\mu$. For example, from eq 6 for $\mathrm{Mg}_{2} \mathrm{Si}$ at $600 \mathrm{~K}$, we have

$$
\mu=-\left(\frac{\partial F}{\partial \epsilon}\right)_{T}=174.336 \epsilon+1.2353 \text {. }
$$




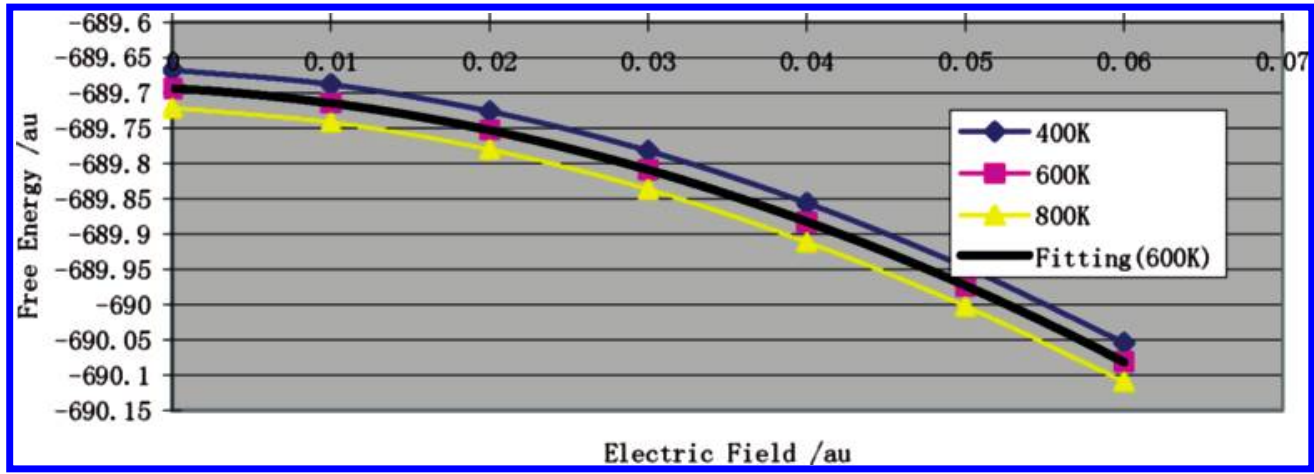

Figure 1. Comparison between fitted functions and numerical data for free energy with respect to the electric field for $\mathrm{Mg}_{2} \mathrm{Si}$ at temperatures of 400,600 , and $800 \mathrm{~K}$.

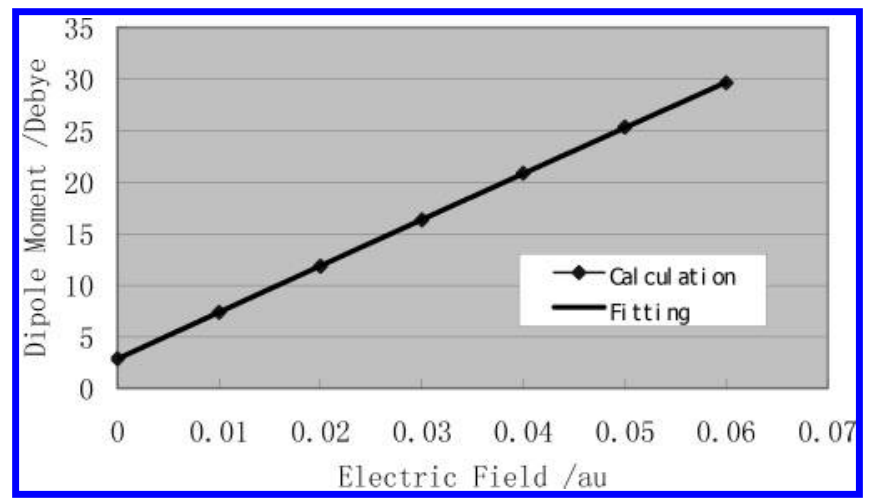

Figure 2. Comparison between the fitted function and numerical data for the dipole moment (Debye) with respect to the electric field (au) for $\mathrm{Mg}_{2} \mathrm{Si}$.

when $\epsilon=1 \mathrm{au}), \mu=175.5713$ Debye. If the free energy is changed from atomic units to the cgs system, that is, ergs, and the electric field is changed from atomic units to cgs-e, we have $\mu=\left(175.5713 \times 4.3598 \times 10^{-11)} /\left(1.714088 \times 10^{7}\right)=446.57\right.$ $\times 10^{-18} \mathrm{cgs}-\mathrm{cm}$.

Because the average dipole moment is numerically equal to polarizability $\alpha$ at the unit electric field, therefore, polarizability $\alpha$ can be calculated as

$$
\begin{aligned}
& \alpha=\left(446.57 \times 10^{-18}\right) /\left(5.142258 \times 10^{9} \times \frac{1}{300}\right)= \\
& 26.0529 \times 10^{-24} \mathrm{~cm}^{3}
\end{aligned}
$$

and inserting the above result into eq 5 leads to the molar polarization,

$$
\psi=\frac{4 \pi}{3} N_{0} \alpha=2.522 \times 10^{24} \times 26.0529 \times 10^{-24}=
$$

$65.7054 \mathrm{~cm}^{3} / \mathrm{mol}$

Similarly, the polarizability $\alpha$ and molar polarization $\psi$ of $\mathrm{Mg}_{2} \mathrm{Si}$ at temperatures from 300 to $800 \mathrm{~K}$ are computed and summarized in Table 2.

C. Distortion Polarizability and Distortion Molar Polarization. In order to calculate the distortion polarizability, we need to fit the data of the average dipole moment (Debye) with electric field in Table 1 into an analytical function by

$$
\mu=-100.77 \epsilon^{2}+453.38 \epsilon+2.8491
$$

Figure 2 shows the fitting is very accurate, and by using eqs 7 and 12 , we have the distortion polarizability.

$$
\alpha_{D}=\frac{\mathrm{d} \mu}{\mathrm{d} \epsilon}=-201.54 \epsilon+453.38
$$

For example, when $\epsilon=1$ (au),

$$
\begin{aligned}
\alpha_{D} & =251.84 \text { Debye } / \mathrm{au}=251.84 \times 10^{-18} \\
& (\mathrm{cgse}-\mathrm{cm}) / 1.714088 \times 10^{7}\left({\left.\mathrm{cgs}-\mathrm{cm}^{-1}\right)}^{-24} \mathrm{~cm}^{3}\right.
\end{aligned}
$$

and from eq 9, distortion molar polarization is calculated as

$\psi_{\mathrm{D}}=\frac{4 \pi}{3} N_{0} \alpha=2.522 \times 10^{24} \times 14.692 \times 10^{-24}=$

$37.054 \mathrm{~cm}^{3} / \mathrm{mol}$

From Table 2, the total polarizability $\alpha$ for $\mathrm{Mg}_{2} \mathrm{Si}$ at $500 \mathrm{~K}$ is $26.105 \times 10^{-24} \mathrm{~cm}^{3}$, and from eq 13 , the distortion polarizability is $\alpha_{D}=14.692 \times 10^{-24} \mathrm{~cm}^{3}$. We can now calculate the orientation polarizability, $\alpha_{0}$, from eq 6 as

$$
\begin{aligned}
\alpha_{0}=\alpha-\alpha_{\mathrm{D}}=(26.105-14.692) \times & 10^{-24} \mathrm{~cm}^{3}= \\
& 11.4131 \times 10^{-24} \mathrm{~cm}^{3} .
\end{aligned}
$$

As we know, atomic polarizability is estimated in the range of $\sim 2.5-5 \%$ of the distortion polarizability, ${ }^{18}$ that is, $(\sim 0.3673-$ $0.7346) \times 10^{-24} \mathrm{~cm}^{3}$. Therefore, electronic polarizability, $\alpha_{\mathrm{E}}$, is given by

$$
\begin{aligned}
& \alpha_{\mathrm{E}}= \alpha_{\mathrm{D}}-\alpha_{\mathrm{A}}=[14.692-(\sim 0.3673- \\
&0.7346)] 10^{-24} \mathrm{~cm}^{3}=(\sim 14.3247-13.9574) 10^{-24} \mathrm{~cm}^{3}
\end{aligned}
$$

D. Dielectric Constant for Solid $\mathbf{M g}_{2} \mathrm{Si}$. Because there are no the translational and rotational motions for solid $\mathrm{Mg}_{2} \mathrm{Si}$, the orientation polarizability of solid $\mathrm{Mg}_{2} \mathrm{Si}$ would be zero $\left(\alpha_{0} \approx\right.$ 0 ), and because the atomic polarization, $\alpha_{\mathrm{A}}$, in the molecule is rather small, it can be reasonably assumed that in the equilibrium of the solid, $\alpha_{\mathrm{A}}$ would be zero. Finally, the total polarizability of solid $\alpha$ will be equal to the electronic polarizability, $\alpha_{E}$, that is, $\alpha \cong \alpha_{\mathrm{E}}=(\sim 14.3247-13.9574) \times 10^{-24} \mathrm{~cm}^{3}$ (from Section III C). Thus, its molar polarization is given by

$\psi=\frac{4 \pi}{3} N_{0} \alpha=2.522 \times 10^{24} \alpha=(\sim 36.13-35.20) \mathrm{cm}^{3} / \mathrm{mol}$ 


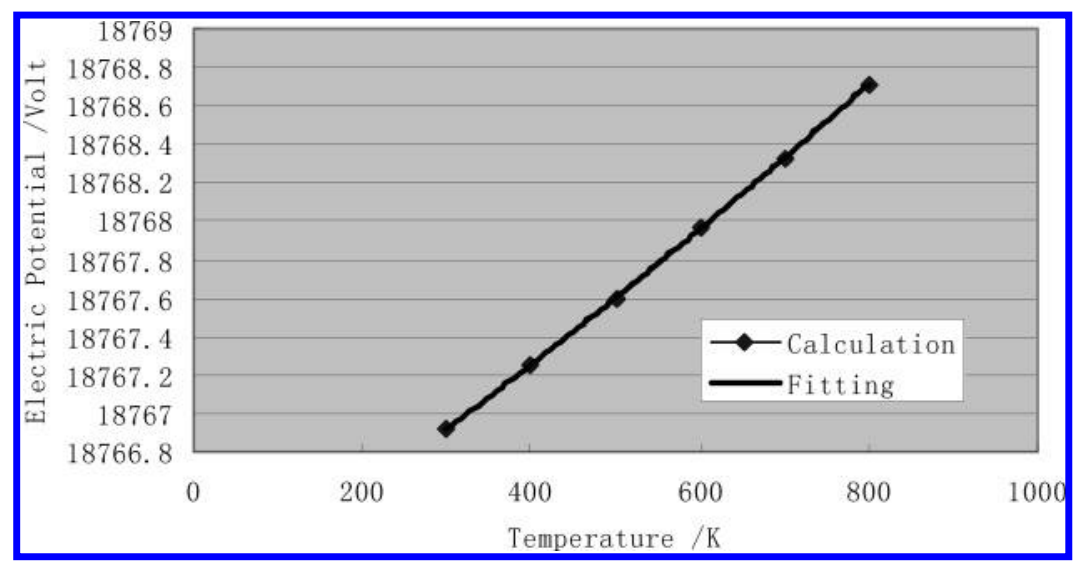

Figure 3. Comparison between the fitted function and numerical data for the electric potential with respect to temperature for $\mathrm{Mg}_{2} \mathrm{Si}$ at zero electric field.

TABLE 3: Free Energy with Respect to Temperature for Crystal $\mathrm{Mg}_{2} \mathrm{Si}$

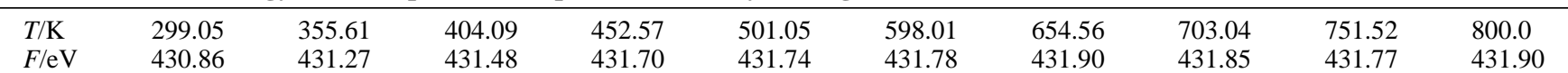

The dielectric constant, $e$, for a pure compound can be calculated from the Clausius-Mossotti formula, ${ }^{7}$

$$
\psi=\frac{e-1}{e+2} \frac{M}{\rho}
$$

Inserting $\psi=(\sim 36.13-35.20) \mathrm{cm}^{3} / \mathrm{mol}$, molecular weight $\mathrm{M}$ $=76.70$, and $\rho=1.88$ into eq 14 leads to the dielectric constant $e=(\sim 24-20)$. This shows that the present calculation is in very good agreement with the experimental value of $e=20 .{ }^{11,12}$

\section{The Thermoelectric Effect and Seeback Coefficient for Solid $\mathrm{Mg}_{2} \mathrm{Si}$}

If the energy gap between the conduction band and the valence band is small or owing to the crystal defect, the electrons can be excited from the valence band to the conduction band by their thermal motion so that it leads to conductivity. The electric potential difference, $\Delta p$, is developed as a result of the temperature difference, $\Delta T .{ }^{13,14}$ This effect is expressed as

$$
S=\lim _{\Delta T \rightarrow 0} \frac{\Delta p}{\Delta T}=\frac{\mathrm{d} p}{\mathrm{~d} T}
$$

which is called the Seebeck coefficient or thermoelectric power. The Seebeck coefficient is conventionally calculated by the carrier motion of the energy band. In the present work, we use a different method that is based on the idea of chemical potential and the thermodynamic method presented in Section II. It is well-known that the Fermi-Dirac distribution can be written as

$$
\begin{array}{r}
N_{i}=\frac{g_{i}}{\exp (\alpha+\beta E+1)}=\frac{g_{i}}{\exp \left[\left(E_{i}-E_{F}\right) / k T\right]+1}= \\
\frac{g_{i}}{\exp \left[\left(E_{i}-\mu\right) / k T\right]+1}
\end{array}
$$

where

$$
\beta=\frac{1}{k T} \quad \text { and } \quad \alpha=-\frac{\mu}{k T}
$$

$N_{i}$ represents the number of electrons in the energy level $E_{i}$ at temperature $T ; E_{F}$ is the Fermi level or simply the chemical potential, $\mu$, of a single electron. ${ }^{15}$ The electron will be spontaneously transferred from the Fermi level region into the lower level of the conduction band district. From thermodynamic relations for an open system or closed system with a charge movable in different energy levels, we have ${ }^{13}$

$$
\mathrm{d} U=T \mathrm{~d} S-P \mathrm{~d} V+\sum \mu_{i} \mathrm{~d} n_{i}+p \mathrm{~d} q+\sigma \mathrm{d} \epsilon+\ldots
$$

where $\sum \mu_{i} \mathrm{~d} n_{i}, v \mathrm{~d} q$, and $\sigma \mathrm{d} \epsilon$ are the chemical work, electric work and surface tensor work done on this system, respectively. If we consider electric work only,

$$
\mathrm{d} U=T \mathrm{~d} S-P \mathrm{~d} V+p \mathrm{~d} q
$$

and for free energy

$$
\mathrm{d} F=\mathrm{d}(U-T S)=-S \mathrm{~d} T-P \mathrm{~d} V+p \mathrm{~d} q
$$

where

$$
S=-\left(\frac{\partial F}{\partial T}\right)_{p, q} \quad P=-\left(\frac{\partial F}{\partial V}\right)_{T, q} \quad \text { and } \quad p=\left(\frac{\partial F}{\partial q}\right)_{T, V}
$$

where the third part of eq 21 that is the partial derivative of free energy with respect to charge represents electric potential. As a general rule, it is called the "chemical potential of charge". In brief, the electric potential is the free energy of unit charge from which we can find the relation between the electric potential and the temperature, and finally, it leads to the Seebeck coefficient evaluated from eq 15.

Now, we use three different methods to calculate the Seebeck coefficient. First, we use the method called molecular structure calculation. The relations of free energy $F(\mathrm{au})$ with respect to both electric field (au) and temperature $(\mathrm{K})$ for $\mathrm{Mg}_{2} \mathrm{Si}$ are listed in Table 1, from which the free energy, $F$, at zero electric field is taken, and its unit is changed from atomic units to electron volts. By using eq 21, the free energy with unit of electron volts is divided by unit electron charge, and we obtain the relation of electric potential with respect to temperature. This function relation can be fitted as follows,

$$
p=\left(6 \times 10^{-7}\right) T^{2}+0.003 T+18766
$$


TABLE 4: The Comparison of Seebeck Coefficient for the Three Methods

\begin{tabular}{|c|c|c|c|}
\hline method & functions of $p \sim T$ & $\begin{array}{l}\mathrm{S} / \mathrm{mV} / \mathrm{K} \\
(300 \mathrm{~K})\end{array}$ & $\begin{array}{l}\mathrm{S} / \mathrm{mV} / \mathrm{K} \\
(800 \mathrm{~K})\end{array}$ \\
\hline 1 & $p=\left(6 \times 10^{-7}\right) T^{2}+0.003 T+18766$ & 3.36 & 3.96 \\
\hline 2 & $p=\left(3 \times 10^{-8}\right) T^{2}+\left(1.5 \times 10^{-4}\right) T+610.47$ & 0.158 & 0.198 \\
\hline 3 & $p=\left(3 \times 10^{-6}\right) T^{2}+0.0018 T-0.3675$ & 3.6 & 6.6 \\
\hline
\end{tabular}

and Figure 3 shows that the fitting is very good. The Seebeck coefficient is obtained by

$$
S=\frac{\mathrm{d} p}{\mathrm{~d} T}=1.2 \times 10^{-6} \times T+0.003
$$

when $T=300 \mathrm{~K}, S=3.36 \mathrm{mV} / \mathrm{K}$, and when $T=800 \mathrm{~K}, S=$ $3.96 \mathrm{mV} / \mathrm{K}$. Because the above relation represents the molecule $\mathrm{Mg}_{2} \mathrm{Si}$ at zero electric field, this should correspond to the gas state. However, the $\mathrm{Mg}_{2} \mathrm{Si}$ material is a polar solid so that the electric potential inside this dielectric should be that of the gas state divided by the calculated dielectric constant of $\sim 24-20$ from the Section III D. This comes to our second method that leads to the function of the electric potential with respect to temperature for solid $\mathrm{Mg}_{2} \mathrm{Si}$. For simplicity, we just divided eq 22 by eq 20 , and then we have

$$
p=\left(3 \times 10^{-8}\right) T^{2}+\left(1.5 \times 10^{-4}\right) T+610.47
$$

when $T=300 \mathrm{~K}, S=0.158 \mathrm{mV} / \mathrm{K}$, and when $T=800 \mathrm{~K}, S=$ $0.198 \mathrm{mV} / \mathrm{K}$.

To compare the above two methods, we perform the third method, which is that the variance of the free energy with respect to temperature is calculated by the energy band structure theory using the "program materials studio 4.0". The calculated results of the free energy with respect to temperature for crystal $\mathrm{Mg}_{2}-$ $\mathrm{Si}\left(\mathrm{Fm} 3 m-\mathrm{O}_{h}^{5}, a=6.338 \AA^{10}\right)$ are given in Table 3, which is used to calculate the polarizability and thermoelectric effects in the following. It is well-known that the energy gap is 0.77 , 0.65 , and $2.1 \mathrm{eV}$ for the electrical, optical, and direct measurement, ${ }^{10}$ respectively. The energy gap from the present calculation is $1.452 \mathrm{ev}$. By Table 3, the function relation of the electric potential with respect to temperature is fitted as

$$
p=\left(3 \times 10^{-6}\right) T^{2}+0.0018 T-0.3675
$$

when $T=300 \mathrm{~K}, S=3.6 \mathrm{mV} / \mathrm{K}$, and when $T=800 \mathrm{~K}, S=$ $6.6 \mathrm{mV} / \mathrm{K}$. All of the results from the above three methods are summarized in Table 4.

Method 2 is in good agreement with the experimental value that shows the Seebeck coefficients are $0.1-0.2$ or $0.24-0.45$ $\mathrm{mv}$ according to the ratio of $\mathrm{Mg}$ and $\mathrm{Si}$ from 300 to $800 \mathrm{~K}^{3}$ and also comparable to the reference value of $0.565 \mathrm{mV} / \mathrm{K} .^{11}$ Method 1 from the gas state and method 3 from the energy band structure theory show similarity, but the two are far from the experimental values.

\section{Concluding Remarks}

Thermodynamics is of sufficient generality to include force fields, such as an electric field, etc. The key to evaluating the Seebeck coefficients is the calculation of the electric potential, from which we have acquired the functions of electric potential with respect to temperature. Then, by usage of the method called molecular structure calculation, we have obtained the average dipole moment that is used to derive the polarizability, $\alpha$, and the molar polarization, $\psi$, of solid $\mathrm{Mg}_{2} \mathrm{Si}$. Finally, it leads to the dielectric constant. The present calculation shows that the dielectric constant of $\sim 24-20$ is in very good agreement with the experimental value of 20 . This accurate dielectric constant plays an essential role in our accurately computing the Seebeck coefficients. The thermodynamic method with molecular structure calculation shows much better results than that of the energy band structure theory using Material studio 4.0.

Acknowledgment. Z. H. Zhu thanks Sichuan University for support, and C. Zhu thanks National Chiao Tunng University for support. This work is supported in part by the National Science Council of the Republic of China under Grant no. 952113-M-009-014.

\section{References and Notes}

(1) Marchuk, N. D.; Zaitsev, V. K.; Fedorov, M. I.; and Kaliazin, A. E. Proceeding of the $8^{\text {th }}$ International Conference on Thermoelectric Conversion 1989, Nacy 210.

(2) Whitsett, C. R.; Danielson, G. C. Phys. Rev. 1955, 100, 1261.

(3) Yoshinaga, M.; Lida, T.; Noda, M.; Endo, T.; Takanashi, Y. Thin Solid Films 2004, 461, 86-89.

(4) Ishii, H.; Matsuo, S.; Karimov, P.; Kawai, J. Phys. Rev. B: Condens. Matter Mater. Phys. 2005, 71, 205202.

(5) Ivanenko, L. I.; Shaposhnikov, V. L.; Filonov, A. B.; Krivosheeva A. V.; Borisenko, V. E.; Migas, D. B.; Miglio, L.; Behr, G.; Schumann, J. Thin Solid Films 2004, 461, 141-147.

(6) Zhang, J.; Fan, Z. J. Mater. Sci. Lett. 2000, 19, 1325-1328.

(7) Lide, D. R. Handbook of Chemistry and Physics; CRC Press LLC: Boca Raton, 2003.

(8) Liu, E. K.; Zhu, B. S.; Luo, J. S. Physics of Semiconductors; Xian Jiao Tong University Press: Xian, P. R. China, 2002.

(9) Samoluoviqi, A. G. Thermodynamics and Statistical Physics (Chinese Translation); High Education Press: Beijing, 1958.

(10) Folland, N. O. Phys. Rev. 1967, 158, 764

(11) Bose, S.; Acharya, H. N.; Banerjee, H. D. J. Mater. Sci. 1993, 28 , $5461-5468$

(12) McWilliams, D.; Lynch, D. W. Phys. Rev. 1963, 130, 2248.

(13) Lay, J. E. Thermodynamics Charles E. Merrill Books, Inc.: Columbus, 1963; pp 607-610.

(14) Alexander, O.; Animalu, E. Intermediate quantum theory of crystalline solids; Prentice Hall: Englewood Cliffs, NJ, 1977; p 242.

(15) Aston, J. G.; Fritz, J. J. Thermodynamics and Statistical Thermodynamics; John Wiley and Sons, Inc.: New York, 1959; pp 353-355.

(16) Morris, R. G.; Rkdin, R. D.; Daniklson, G. C. Phys. Rev. 1958, 109, 1909-1915.

(17) Xie, A. D.; Yan, S. Y.; Zhu, Z. H.; Fu, Y. B. Chin. Phys. 2005, 14, 1808-1805

(18) Wheatley, P. J. The Determination of Molecular Structure; Clarendon Press: Oxford, 1959; p 208. 